\title{
Annual Spring Forum Held at NAS
}

\author{
Elton N. Kaufmann*
}

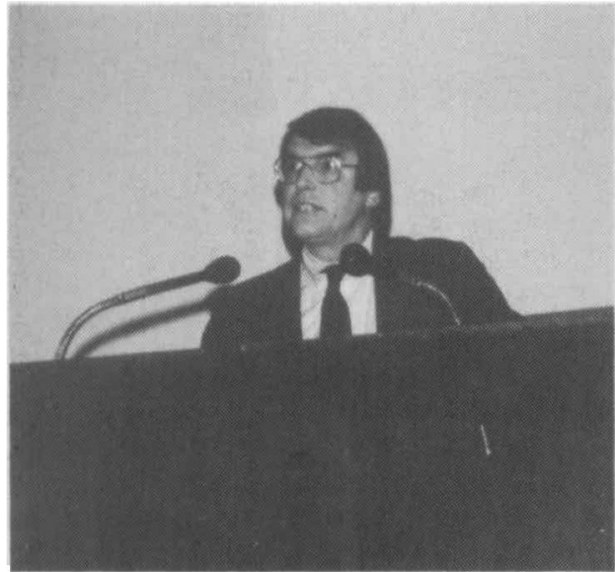

George A. Keyworth (chairman of Keyworth/Meyer International and former science advisor to President Reagan) speaks on the state of science in America.

The Solid State Sciences Committee (SSSC) and the Committee on Atomic and Molecular Science (CAMS) held their joint spring forum on March 10 and 11, 1986 at the National Academy of Sciences in Washington, DC. Presentations covered both science policy and funding issues as well as forefront areas of basic research. After introductory remarks by Al Narath and Lloyd Armstrong, who are respectively chairmen of the SSSC and CAMS, the first session comprised talks by Harold Hanson, Executive Director for the House Science and Technology Committee; Hugh Loweth of the Office of Management and Budget (OMB); Richard Nicholson, Assistant Director for Mathematical and Physical Sciences at the National Science Foundation; Leo Young, Director for Research and Laboratory Management at the Department of Defense; and Alvin Trivelpiece, Director of the Department of Energy's Office of Energy Research.

Hanson summarized the current budget situation, emphasizing that things are in a state of flux and eventual outcomes are uncertain. The generally austere picture for basic research was confirmed, however. In answer to a question from Herbert Johnson (Cornell University). Hanson noted that the direct "pork barrel" approach of universities to congress for funds "is not a new phenomenon." What's new is that "they are now using public relations firms and professional fund raisers called 'vice presidents." Hanson noted that Congressman Fuqua (Florida) is at tempting to create "a proper path for construction funds requests" but he held out little hope for such a process in a "Gramm-Rudman era." Concerning classified research on campuses-a point raised by I. Sellin (Oak

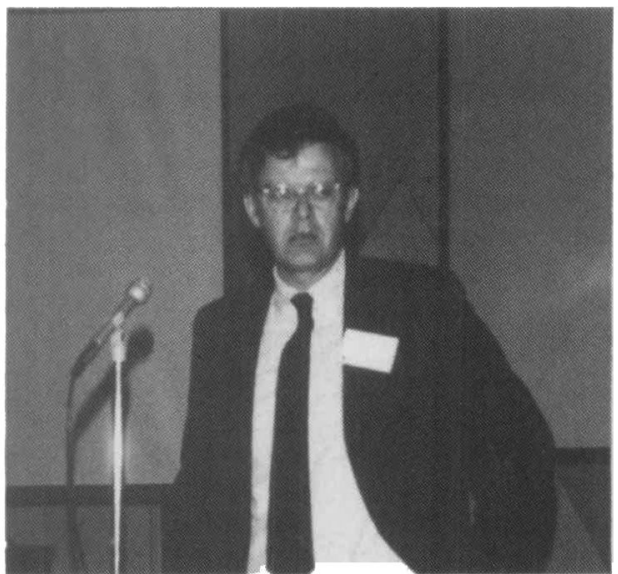

John M. Poate (A T\&T Bell Labs and former MRS president) reports on an NMAB study of "Electronic Materials and Surface Modification in the United States and Japan."

Ridge National Laboratory)-Hanson favored both openness in research and the right of researchers to study what they want, including classified programs, but reminded the audience that his House Committee does not deal with DOD-sponsored research.

Loweth echoed the combination of austerity and uncertainty in present budget deliberations. He also informed the group that $O M B$ is sensitive to the issue of large facility versus small science. In answer to a query from Morris Cohen (MIT) he noted that increased funds to NSF explicitly recognize the importance of small science. He continued by noting that one large facility, the proposed space station, is not viewed in the same context by the administration or congress and is thus not in a "tradeoff" relationship with other science support Loweth conceded that such programs as the cold-neutron source at NBS are having a tough time getting support in spite of their clear desirability. In answer to a question from Klaus Zwilsky of the $\mathrm{Na}$ tional Research Council (NRC), another $\mathrm{OMB}$ representative in the audience, Judith Bostick, indicated that OMB intends to keep the input from the NRC separate from that of the three-person council mandated by the National Critical Materials Act of 1984

Nicholson of NSF noted that NSF funding had enjoyed recent healthy advances and is now contracting in direct response to Gramm-Rudman mandates. After covering some of the percentage decrements various programs will suffer, he noted that NSF may revise policies toward principal investigator summer salary support and toward indirect costs charged against research grants. Leo Young (DOD) empha-

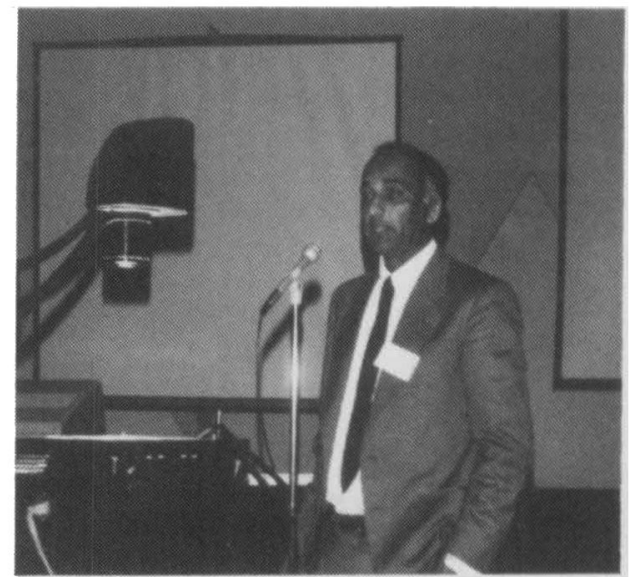

Praveen Chaudhari (IBM), co-chair of the Materials Science and Engineering Study, announces chairpersons for the Study's five panels.

sized in his remarks the new DOD "University Research Initiative." He said that the requested budget for the support of human resources, instrumentation and multidisciplinary research programs was $\$ 25$ million in fiscal year 1986 followed by $\$ 50$ and $\$ 100$ million for 1987 and 1988, respectively. The pre-Gramm-Rudman congressional reaction was to allocate $\$ 100$ million for the first year (now about $\$ 90$ million due to cuts) reflecting strong legislative support in the House Armed Services Committee for a faster implementation of the initiative. (Also during the week of March 10, concerns were expressed in testimony to that committee by the new Deputy Under Secretary of Defense for Research and Technology, Ronald Kerber, that the merit-based awards intent was in danger of being subverted by earmarking of funds in the House Appropriations Committee.)

In the final remarks of the first morning of the forum, Alvin Trivelpiece of DOE offered several pointed comments to emphasize the linkage between funding decisions and political considerations. He contended that the short-lived National Center for Advanced Materials (NCAM) initiative at Lawrence Berkeley Laboratory "would have died even without bad mail" in the present policy and budget climate. In noting that LBL is associated with the current FY87 funding of the $1-2 \mathrm{GeV}$ synch rotron, he pointed out that the SeitzEastman report which put the $6 \mathrm{GeV}$ machine as a higher priority was advice from the Academy and not binding in the sense that an Energy Research Advisory Board (ERAB) review would be. He further mentioned the $6 \mathrm{GeV}$ machine destined for

continued 
Argonne National Laboratory, the relativistic heavy-ion collider for Brookhaven National Laboratory, and a high-flux reactor at Oak Ridge National Laboratory, all, of course, subject to funding.

Trivelpiece admonished the audience by claiming that they failed to recognize the connection between these projects and (political) events. The SSC (superconducting supercollider) is part of the equation too, he noted. "Attempts to kill one equals an at tempt to kill all collectively," according to Trivelpiece. "If you shoot the other guy in the foot, you'll be limping right along with him." As an example of the unfortunate funding and priority algorithms now he noted that the $\$ 350$ million MFTF$B$ fusion research facility at the Lawrence Livermore Laboratory was mothballed shortly after its dedication because of a $\$ 10$ to $\$ 15$ million shortfall "at the margin" to avoid spreading the cuts around all fusion projects. In answer to a final question relating all these large facility issues to small science support, Trivelpiece expressed the belief that "the general climate causes small and big science to track each other," implying a coat tail effect is operative.

The afternoon was devoted to four presentations covering state-of-the-art techniques for surface and molecular science. Leading off was Jene Golovchenko (AT\&T Bell Laboratories) who described tunneling microscopy. He demonstrated how computer processed images derived from the signals needed to maintain tunneling electrode-to-surface distance can appear as though one were viewing actual arrays of individual atoms (or as Golovchenko picturesquely calls them, "puff balls"). Next to speak was David Weinland (NBS, Boulder, CO) who described advanced techniques used to trap particles for sufficiently long times to allow extremely accurate spectroscopic measurements. Not only the use of electromagnetic fields and field gradients were described (the latter being necessary for neutral particles when magnetic moments interact with the fields), but a method based on directional photon absorption followed by isotropic emission using multiple laser beams was detailed. Advances in lasers with regard to ultrashort pulses were next revealed by Daniel Grischkowsky (IBM) who showed how a variety of techniques have led to pulse widths of tens of femtoseconds. One aspect of the process of bunching the light pulse has been given the acoustic-like label, "chirping the pulse," by which is meant passing the pulse through a nonlinear medium which broadens its wavelength content so that a second dispersive medium can overlap the various frequency components in time. The applications of short pulses to molecular science were then presented by Ahmed Zewail (Caltech).

Day two of the joint forum was led off by an upbeat address by George A. Keyworth, former Science Advisor to the President.
Rather than characterizing the state of science in America as in trouble, he views it as extremely healthy and believes "now is not a period of austerity but of opportunity." He regards "mutually reinforcing disciplines" as the unique aspect of the 1980 s and cites the "flowering of materials science" as an example. Not just multidisciplinary, but multi-institutional, collaborations lead to success; that is, joint efforts of universities, industry and federal labs are symptoms of the healthy situation (See Material Matters in this issue.)

Keyworth regards the advent of the NSF's Engineering Research Centers as "the single most important initiative in the last five years." He pointed to the "ease with which one field uses the tools and insights of others" as one reason for the current rapid advancement of all fields of science. Somewhat reminiscent of the prior day's comments by Trivelpiece, Keyworth said that, "there is no questioning in government of the general idea that research in science is valuable (and) whenever the science community has been able to get its act together and present a coherent program to the Congress, we have made progress." It was Keyworth's hope that his proposal for a cabinet-level Department of Science and Technology would provoke useful debate in this area

Speaking to the trends, past and present, in physics manpower and funding, Daniel Kleppner (MIT) drew a stark distinction between the overall positive situation described by Keyworth and emphasized the serious lack of support in some areas such as atomic, molecular and optical physics research. Trends displayed by Kleppner emphasized the drop-off in resources in many areas over the last decade and more. By inference, he indicated that support was preferentially going to the more applied fields.

Returning again to technical presentations, John Silcox (Cornell University) and Gary Kellogg (Sandia National Laboratories) spoke on high-resolution electron microscopy and on atom-probe and fieldion microscopy, respectively. In both cases, impressive advances were detailed wherein atomic level resolution with compositional sensitivity is available. Silcox emphasized significant advances in technique to overcome electron lens aberrations and also stated that the frontier of the technique is in computer image processing, storage, and calculation and automated instrument control. Kellogg explained the extension of the well-known field-ion microscope to composition analysis by mean of a time-offlight atom probe and to application to insulators, ceramics, and glasses by replacing the high-voltage pulse used for atom removal with a laser pulse. The combined impression gleaned from the technical talks of both days was that the materials characterization field has made huge strides in directions that will serve microscopic understanding of structures and properties well. Although strides of equal magnitude have yet to surface in areas such as materials processing or synthesis, the speakers hinted at capabilities to perform atom-by-atom surface engineering as possibilities for the future.

After presentations by Armstrong and Narath on the work of the CAMS and SSSC, respectively, John Poate (AT\&T Bell Laboratories, and former president of MRS) gave a preliminary report on the study conducted by a committee of the National Materials Advisory Board of the National Academy of Engineering concerning "Electronic Materials and Surface Modification in the United States and Japan." Poate noted several areas of semiconductor processing where the United States lags. The committee, which visited several industrial and some academic institutions in Japan, is expected to issue its final report in April.

To end the forum, the schedule included a review of the results of the ERAB Panel on Materials Facilities by Donald Stevens (DOE) and a status report from Praveen Chaudhari (IBM) on the Materials Science and Engineering Study just under way under the auspices of the SSSC and NMAB and managed by NRC's Board on Physics and Astronomy. Chaudhari, who co-chairs the Study with Merton Flemings (MIT), announced their intention to complete the bulk of the Study's work within one year. As described in the January/February issue of the BULLETIN (Vol. XI, No. 1, p. 63), the study consists of five panels. Each panel is led by a chairperson and two vice chairpersons who were named as follows by Chaudhari during his presentation:

1. "Materials Research Opportunities and Needs in MS\&E." J. Langer (UCSB), G. Parshall (Dupont). J. Stiegler (ORNL)

2. "Exploitation of MS\&E and Technology for National Welfare." A. Chynoweth (Bell Communications Research), R. G. Kepler (Sandia National Laboratories), J. C. Williams (Carnegie-Mellon University)

3. "International Cooperation and Competition." L. Schwartz(NBS), W. D. Compton (NAE), R. Roy (Pennsylvania State University)

4. "Research Resources in MS\&E." $T$. Loucks (Norton Co.), M. Blume (Brookhaven National Laboratory), G. Whitesides (Harvard University)

5. "Education in MS\&E."). Cohen (Northwestern University), R. Hansen (AmesDOE), J. Hulm (Westinghouse).

Narath closed the proceedings with comments regarding his intention to look into ways to revitalize the operations of the SSSC, particularly with regard to improving communication with and attendance by the research public which the Committee intends to serve.

"Elton Kau/mamn, of Lawrence Livermore National Laboratory, is past president of the Materials Research Society. 